\title{
Le thorium : analyse de la réglementation française
}

\author{
P. LAROCHE*, P. GERASIMO**
}

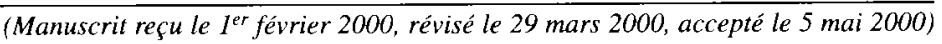

RÉSUMÉ La réglementation applicable au thorium a été analysée, car elle peut, dans certaines circonstances, prêter à confusion. L'expression « thorium naturel » étant réservée à l'association de deux émetteurs alpha (le thorium 232 et le thorium 228), cette définition ne prend pas en compte les émetteurs bêta et gamma de la filiation du ${ }^{232} \mathrm{Th}$. L'ambiguïté est majorée par le fait qu'il n'existe pas de différence entre le thorium naturel et le thorium utilisé dans l'industrie. La nouvelle réglementation, découlant de la directive européenne 96-29, prend en compte la radioactivité des émetteurs naturels et devrait permettre une évolution dans la protection des travailleurs.

ABSTRACT Thorium: an analysis of the existing French regulation.

We carefully surveyed the regulation effective for thorium, which in some cases, may be a source of misunderstanding. First, the phrase "natural thorium" defines the coupling of two alpha emitters $\left({ }^{232} \mathrm{Th}\right.$ and $\left.{ }^{228} \mathrm{Th}\right)$ and it does not include the beta and gamma emitters originating from the ${ }^{232} \mathrm{Th}$. Further confusion may arise from the fact that there is no difference between natural thorium and thorium used in industry. The new regulation, deriving from the European directive (number 96-29), takes into account the radioactivity of natural emitters and should, in that respect, guarantee an improvement in the safety of working conditions.

\section{Définition}

L'incertitude sur la relation causale aux faibles doses à conduit à établir la réglementation sur la base d'une relation linéaire et sans seuil. Dans cette hypothèse, elle contribue à limiter les atteintes à la santé de deux catégories de personnes, publics et travailleurs. Elle doit permettre une protection adéquate contre les rayonnements ionisants tout en évitant d'entraver les activités industrielles. La diversité et le nombre élevé des utilisations ainsi que l'analyse des risques professionnels montrent une possibilité réelle d'exposition interne ou externe chez les travailleurs de la filière thorium (Laroche, 1998a), il convient donc de vérifier si la réglementation applicable au thorium est adaptée.

* Service médical de la base de l'Ile Longue, BP N ${ }^{\circ} 50029240$ Brest Naval, France.

** Laboratoire de contrôle radiotoxicologique, SPRA BP N ${ }^{\circ} 100480$ Ammées, France. 


\section{La réglementation actuelle}

Les textes réglementaires qui régissent, en France, la radioprotection sont une transposition des recommandations et réglementations adoptées au niveau international et européen. La réglementation actuelle découle des recommandations de la Commission internationale de protection radiologique (CIPR). La CIPR formule des recommandations essentiellement fondées sur les rapports de l'UNSCEAR (Comité scientifique des Nations Unies sur les effets des rayonnements ionisants, créé en 1955), du National Research Council et sur les études radiobiologiques fondamentales du comité BEIR (Biological Effects of Ionizing Radiation).

Ces recommandations de la CIPR ont fortement influencé les normes fondamentales de radioprotection adoptées par l'Agence internationale de l'énergie atomique (AIEA, fondé en 1955) ainsi que les directives de la Communauté européenne de l'énergie atomique (CEEA ou EURATOM, fondée le 25 mars 1957).

La réglementation française actuelle ainsi que certaines modifications du code de la santé et du code du travail sont les conséquences de la transposition de la directive EURATOM du 15 juillet 1980, modifiée le 3 septembre 1984. L'adoption de la nouvelle directive EURATOM du 13 mai 1996, fixant les normes de base relatives à la protection sanitaire de la population et des travailleurs contre les dangers résultant des rayonnements ionisants, va entraîner, prochainement, une révision de la réglementation française.

La production, le traitement, la manipulation, l'utilisation, la détention, le stockage, le transport et l'élimination de matériaux contenant du thorium, doivent se conformer à plusieurs dispositions légales et réglementaires (Tab. I).

\section{Analyse de la réglementation}

La réglementation actuelle est ambiguë et son analyse provoque bien des commentaires. Trois points sont plus particulièrement à mettre en exergue.

\subsection{La définition du thorium naturel}

Le thorium naturel n'est pas considéré, sous l'angle réglementaire, comme un mélange de substances radioactives (décret $n^{\circ} 66-450$ du 20 juin 1966). L'expression «thorium naturel » est réservée à l'association de deux émetteurs alpha en filiation, les isotopes radioactifs ${ }^{232} \mathrm{Th}$ et ${ }^{228} \mathrm{Th}$. On admet qu'un curie de thorium naturel correspond à $3,7 \times 10^{10}$ désintégrations par seconde, c'est à dire $3,7 \times 10^{10} \mathrm{~Bq}\left(1,85 \times 10^{10} \mathrm{~Bq} \mathrm{de}{ }^{232} \mathrm{Th}\right.$ et $1,85 \times 10^{10} \mathrm{~Bq}$ de $\left.{ }^{228} \mathrm{Th}\right)$. On peut donc écrire l'égalité suivante, $1 \mathrm{~d} \alpha \mathrm{ps}^{*}$ de thorium naturel $=0,5$ dops de ${ }^{232} \mathrm{Th}+0,5$ d $\alpha$ ps de ${ }^{228} \mathrm{Th}$.

* désintégration alpha par seconde. 
TABLEAU I

Législation et réglementation applicable au thorium.

Regulation effective for thorium.

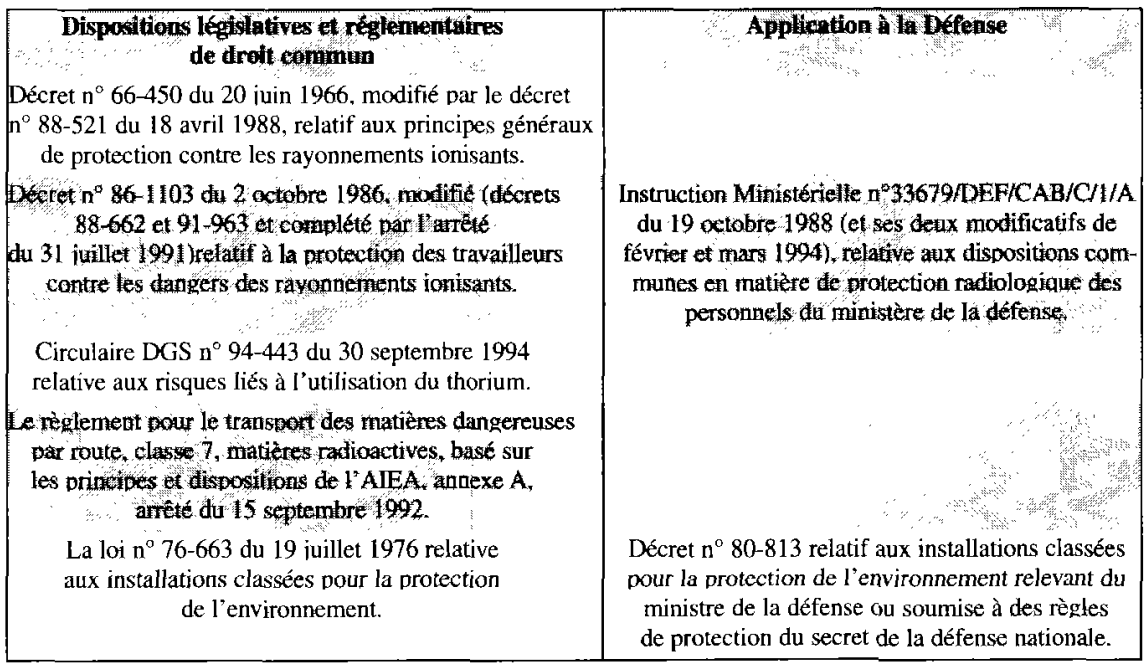

Cette définition ne repose sur aucun critère de physique nucléaire. Le thorium naturel est, en masse, composé presque exclusivement de ${ }^{232} \mathrm{Th}$, ce radioélément étant le chef d'une des trois familles radioactives naturelles, il est théoriquement toujours en équilibre séculaire avec les onze descendants composant sa filiation. Cette équation prend en compte tous les émetteurs alpha, qu'elle assimile à du thorium. Elle ne prend pas en compte les émetteurs bêta ou gamma de la filiation du ${ }^{232} \mathrm{Th}$, ainsi que ceux du ${ }^{230} \mathrm{Th}$, pourtant constamment présents dans le «thorium industrialisé », (Laroche, 1998b) provenant d'une autre famille radioactive. Le calcul, qui consiste à déduire l'activité globale à partir de la masse de ${ }^{232}$ Th (deuxième partie de l'égalité), ne prend en compte que deux émetteurs alpha sur les six composants la filiation. Cette situation provoque une surveillance inadaptée des risques ainsi qu' une mauvaise évaluation des doses éventuelles.

\subsection{L'activité autorisée du thorium naturel}

La législation et l'activité autorisée aujourd'hui pour le thorium sont beaucoup moins restrictives que la législation et les activités appliquées aux radionucléides artificiels, cette situation est générale à tous les radioéléments qualifiés de «naturels». Le thorium n'est pas obligatoirement soumis à un régime de déclaration ou d'autorisation préalable : 
- si son activité est inférieure à 500 kilobecquerels par kilogramme;

- si l'activité totale est inférieure à 50 kilobecquerels ou 5 kilobecquerels respectivement pour les radioéléments de forte toxicité et de très forte toxicité.

(Décret $\mathrm{n}^{\circ} 88-521 \mathrm{du} 18$ avril 1988).

Alors que, paradoxalement, le ${ }^{232} \mathrm{Th}$ et le thorium naturel sont classés dans le groupe II (forte toxicité) et le ${ }^{228} \mathrm{Th}$ dans le groupe I (très forte toxicité). Les limites annuelles d'incorporation (LAI) des isotopes du thorium sont parmi les plus faibles de l'ensemble de la réglementation actuelle (Tab. II). On peut constater que la radiotoxicité des isotopes du thorium est loin d'être négligeable devant celle d'autres radionucléides tels que: ${ }^{137} \mathrm{Cs},{ }^{131} \mathrm{I},{ }^{239} \mathrm{Pu},{ }^{235} \mathrm{U}$ ou ${ }^{238} \mathrm{U}$.

\subsection{Installations classées pour l'environnement}

La loi 76-663 du 19 juillet 1976 relative aux installations classées pour la protection de l'environnement (ICPE) met en place un régime particulier de déclaration et d'autorisation pour les installations utilisant ou éliminant un certain niveau d'activité. Un avis du conseil d'État en date du 23 octobre 1991 édicte

\section{TABLEAU II}

Limites annuelles d'incorporation (LAI en Bq) pour l'exposition professionnelle à différents radioléléments, d'après le décret 86-1103 du 2 octobre 1986.

Annual limit of incorporation (LAI in Bq) for occupational exposition from French decree edicted on the $2 d$ of October 1986.

\begin{tabular}{|c|c|}
\hline radidatnents & Whalation \\
\hline LAI ${ }^{137} \mathrm{Cs}$ & $6 \times 10^{6}$ \\
\hline A A I $1310^{6}$ & $2 \times 10^{6}$ \\
\hline LAI ${ }^{228} \mathrm{Th}$ & $4 \times 10^{2(a)}$ \\
\hline $\mathrm{EAI}^{230 \mathrm{TH}} \quad \mathrm{l} \times 10^{5}$ & $2 \times 10^{2}$ (4) \\
\hline LAI ${ }^{232} \mathrm{Th}$ & $4 \times 10^{1(a)}$ \\
\hline LAI ${ }^{230} 7 \mathrm{~d}$ & $5 \times 10^{2}($ of \\
\hline $5 \times 10^{5(\mathrm{e})}$ & $5 \times 10^{4(g)}$ \\
\hline LAI HO $\quad 5 \times 10^{5}$ & $5 \times 10^{4}(g)$ \\
\hline
\end{tabular}

a/ Tous les composés du thorium ou du plutonium, sauf ceux qui sont indiqués à la note b.

b/ Oxydes et hydroxydes.

c/ Tous les composés du plutonium, sauf $\mathrm{PuO}_{2}$.

$\mathrm{d} / \mathrm{PuO}_{2}$.

e/ Composés inorganiques de l'uranium solubles dans l'eau (uranium hexavalent).

f/ Composés relativement insolubles tels que $\mathrm{UF}_{4}, \mathrm{UO}_{2}, \mathrm{U}_{3} \mathrm{O}_{8}$ dans lesquels l'uranium est habituellement trétravalent.

$\mathrm{g} / \mathrm{UF}_{6}, \mathrm{UO}_{2} \mathrm{~F}_{2}, \mathrm{UO}_{2}\left(\mathrm{NO}_{3}\right)_{2}$.

h/ $\mathrm{UO}_{3}, \mathrm{UF}_{4}, \mathrm{UCL}_{4}$.

i/ $\mathrm{UO}_{2}, \mathrm{U}_{3} \mathrm{O}_{8}$. 
un régime particulier de prise en compte de la radiotoxicité du thorium naturel et de l'uranium naturel:

"L'administration est fondée à ne pas prendre en compte dans le calcul de l'activité totale les produits dont l'activité massique est inférieure aux seuils déterminés dans les conditions prévue par l'article 3 et par l'annexe II du décret du 20 juin 1966 modifié.

L'administration est fondée à ne tenir compte que de l'activité de ces deux éléments (thorium et uranium) dits "têtes de série» à l'exclusion de celles de leurs produits de filiation. »

Cet avis ne tient pas compte des risques mis en évidence au cours de notre étude (Laroche, 1998c) ni de la physique nucléaire. Le thorium utilisé dans l'industrie a été broyé, concassé et purifié chimiquement. L'équilibre séculaire a été rompu et on ne se trouve plus devant une substance naturelle solide, mais devant une matière industrialisée. Ne prendre en compte que les éléments têtes de série et non pas les produits de filiation revient à minimiser considérablement les incorporations éventuelles. De plus la présence du ${ }^{230}$ Th dans les alliages industrialisés est constante. Cet élément est un descendant de $\mathrm{l}^{238} \mathrm{U}$ qui, lui, a été éliminé chimiquement. Aux termes de l'avis du conseil d'État, cet «orphelin de père» ne doit pas être pris en compte.

Cette situation, ambiguë, devrait rapidement trouver sa conclusion. En effet la directive européenne 96/29/Euratom du Conseil du 13 mai 1996 (fixant les normes de base relatives à la protection sanitaire de la population et des travailleurs contre les dangers résultant des rayonnements ionisants) applicable avant le 13 mai 2000, prend en compte dans son titre VII, l'augmentation notable de l'exposition due aux sources naturelles de rayonnement. Le titre VII s'applique en outre aux activités professionnelles au cours desquelles la présence de sources naturelles de rayonnement entraîne une augmentation notable de l'exposition des travailleurs (art. 40). Les activités concernées sont : - les travailleurs ou le public exposés aux produits de filiation du thoron ou du radon; - les activités professionnelles impliquant l'emploi ou le stockage de matières ; contenant des radionucléides, provoquant une augmentation notable de l'exposition des travailleurs ou du public ;

- les activités professionnelles entraînant la production de résidus, non considérés habituellement comme radioactifs (la prise en compte de ces résidus conduirait à transformer plusieurs dépôts importants de résidus minéraux en installations nucléaires de base);

- l'exploitation des avions.

\section{Conclusion}

La réglementation concernant le thorium peut prêter à confusion. L'expression «thorium naturel » étant réservée à l'association de deux émetteurs alpha, le ${ }^{232} \mathrm{Th}$ et 
le ${ }^{228}$ Th. Cette définition ne prend pas en compte les émetteurs bêta et gamma de la filiation du ${ }^{232} \mathrm{Th}$, alors que le thorium naturel est en masse composé presque exclusivement de ${ }^{232} \mathrm{Th}$.

L'ambiguîté de la réglementation est majorée par le fait qu'il n'existe pas de différence entre le thorium naturel et le «thorium industrialisé ». Le thorium naturel n'est pas considéré comme un mélange de substances radioactives, alors que le ${ }^{230} \mathrm{Th}$, provenant de la famille de $\mathrm{l}^{, 238} \mathrm{U}$ est constamment présent dans le «thorium industrialisé ». Plutôt que de continuer à considérer le thorium comme une substance radioactive naturelle solide, il faudrait le considérer comme un produit industriel et lui appliquer un régime de déclaration ou d'autorisation préalable adéquat. Ce radioélément, très radiotoxique et employé dans l'industrie, après avoir été broyé, concassé, traité par un ajout de produits chimiques et enfin concentré, n'a plus rien de naturel. La directive européenne 96-29 du 13 mai 1996 en tenant compte de la radioactivité des émetteurs naturels, prend en considération le problème du thorium. La nouvelle réglementation nationale découlant de cette directive devrait donc permettre d'évoluer dans le sens de la protection sanitaire de la population et des travailleurs exposés aux rayonnements ionisants.

\section{RÉFÉRENCES}

Décret $n^{\circ} 66-450$ du 20 juin 1966, modifié par le décret $n^{\circ} 88-521$ du 18 avril 1988, relatif aux principes généraux de protection contre les rayonnements ionisants.

Décret $n^{\circ} 81-512$ du 12 mai 1981, relatif à la protection et au contrôle des matières nucléaires.

Décret $n^{\circ} 88-521$ du 18 avril 1988 , modification du décret $n^{\circ} 66-450$ du 20 juin 1966 relatif aux principes généraux de protection contre les rayonnements ionisants. J.O. du 6 mai 1988.

Directive 80/836/EURATOM du 15 juillet 1980, modification des directives fixant les normes de base relatives à la protection sanitaire de la population et des travailleurs contre les dangers résultant des rayonnements ionisants. J.O.C.E. du 17 septembre 1980.

Directive 96/29 EURATOM du conseil du 13 mai 1996, fixant les normes de bases relatives à la protection sanitaire de la population et des travailleurs contre les dangers résultant des rayonnements ionisants. J.O.C.E., 29 juin 1996.

Laroche P., Cazoulat A., Lecouteulx I., Schoulz D., Gérasimo P. (1998a) Le thorium : Cet élément oublié de la radioprotection, Archives des Maladies Professionnelles, 59, 7, 469-479.

Laroche P., Cazoulat A., Rotger C., Petitot F., Gérasimo P. (1998b) Le thorium : analyse et dosimétrie des électrodes de soudures thoriées, Ann. Pharmaceutiques française, 56, 3, 123-133.

Laroche P. (1998c) Le thorium: Risques pour les travailleurs surveillance et radioprotection. Thèse de doctorat, université Paris V, Cochin - Port-Royal. 